\title{
Las elecciones de 2018 en México y el triunfo del Movimiento de Regeneración Nacional (Morena)
}

\author{
Jaime Aragón Falomir (México)* \\ Alfredo Edmundo Fernández de Lara Gaitán (México)** \\ Juan Bautista Lucca (Argentina) $)^{* * *}$
}

\section{Resumen}

Este artículo analiza las elecciones de 2018 en México, en las que por primera vez un candidato de un partido de izquierda ganó la Presidencia de ese país, obteniendo una amplia mayoría en el Poder Legislativo en los ámbitos estatal y municipal. Para ello se consideran las dinámicas y patrones que el sistema político mexicano ha presentado en las últimas cuatro décadas a partir de una clave político-histórica. Se analiza el surgimiento del Movimiento de Regeneración Nacional (Morena) como un partido nuevo con capacidad de éxito electoral en este marco de estabilidad partidaria. Se ausculta el pulso político partidario en las campañas electorales para ver cómo se producía la tensión entre los candidatos y las siglas partidarias. Se comparan los resultados electorales de 2006, 2012 y 2018 por circunscripción, para presentar las transformaciones que llevaron al triunfo de Morena. En definitiva, este derrotero permitirá analizar en qué medida la elección [286] en 2018 de un nuevo partido produjo una transformación histórica que inaugura un nuevo sistema de partidos en México.

\section{Palabras clave}

Elecciones; Partidos Políticos; Cambio Político; Movimiento de Regeneración Nacional (Morena); Andrés Manuel López Obrador; México.

Fecha de recepción: agosto de 2018 • Fecha de aprobación: octubre de 2018

\footnotetext{
* Licenciado en Relaciones Internacionales. Magíster en Estudios Internacionales y Europeos (América Latina). Magíster en Investigación en Ciencias Políticas. Doctor en Ciencias Políticas y Sociología: Estudios Latinoamericanos. Profesor e investigador de la Universidad Nacional de Rosario, Consejo Nacional de Investigaciones Científicas y Técnicas (Conicet), Instituto de Investigaciones, Facultad de Ciencias Políticas y Relaciones Internacionales, Universidad Nacional de Rosario, Argentina. Correo electrónico: jaime.aragonf@gmail.com Orcid: https://orcid.org/0000-0003-1706-1259

** Licenciado en Relaciones Internacionales. Magíster en Políticas Públicas Comparadas. Universidad de los Andes, Colombia. Becario Colciencias Doctorado Nacional 727. Correo electrónico: alffer@ gmail.com Orcid: https://orcid.org/0000-0002-0074-3037

${ }_{* * *}^{*}$ Licenciado en Ciencia Política. Magíster en Estudios Latinoamericanos. Doctor en Ciencias Sociales. Profesor e investigador de la Universidad Nacional de Rosario, Consejo Nacional de Investigaciones Científicas y Técnicas (Conicet), Argentina. Correo electrónico: juanlucca@hotmail. com Orcid: https://orcid.org/0000-0001-9017-8619
} 


\title{
Cómo citar este artículo
}

Aragón Falomir, Jaime; Fernández de Lara Gaitán, Alfredo Edmundo y Lucca, Juan Bautista. (2019). Las elecciones de 2018 en México y el triunfo del Movimiento de Regeneración Nacional (Morena). Estudios Políticos (Universidad de Antioquia), 54, pp. 286-308. http://doi.org/10.17533/udea.espo.n54a14

\section{The 2018 Elections in Mexico and the Triumph of the National Regeneration Movement (MORENA)}

\begin{abstract}
This article analyzes Mexico's 2018 elections, in which, for the first time, a leftist party won the presidency, as well as a large majority of the legislative branch both at the federal and at the local level. From a political-historical perspective, first, this study takes into account the dynamics and patterns that the Mexican political system has presented over the past four decades. Second, it analyzes the emergence of the National Regeneration Movement (MORENA) as a new party capable of electoral success in the framework of party stability. Third, it evaluates the party's political pulse in the electoral campaigns to see how the tension between candidates and parties occurred. Fourth, it compares the electoral results of the presidential elections of 2006 and 2012, in order to analyze the historically situated explanations of MORENA's victory. This analytical approach allows to determine to what extent the election of a new party in 2018 produced a historical transformation that established a new political system in Mexico.
\end{abstract}

\section{Keywords}

Elections; Political Party; Political Change; National Regeneration Movement (MORENA); Andrés Manuel López Obrador; Mexico. 


\section{Introducción}

Luego de dos intentos infructuosos por obtener la Presidencia de México — 2006 y 2012 - el otrora miembro tanto del Partido Revolucionario Institucional (PRI) y fundador del Partido de la Revolución Democrática (PRD), Andrés Manuel López Obrador (AMLO), consiguió un triunfo inusitado en 2018 mediante su nueva fuerza partidaria, el denominado Movimiento de Regeneración Nacional (Morena). Estos comicios se destacaron por ser los más grandes en cuanto al número de votantes empadronados, así como de cargos en disputa, y por tener los altos niveles de violencia política, a causa de homicidios a candidatos.

La alianza Juntos Haremos Historia, ${ }^{1}$ encabezada por AMLO, obtuvo una extensa victoria en las elecciones - presidencial, legislativa, estatal y municipal-, al grado de cosechar un total de votos superior a los logrados en conjunto por los otros tres contendientes presidenciales. El triunfo de AMLO fue contundente en todo México, perdiendo únicamente en un estado Guanajuato-, e inclusive en la disputa por el Legislativo federal, donde Morena se erigió como la principal fuerza en ambas cámaras. Estas elecciones son históricas, no solo por la magnitud del resultado, sino por cuestiones

[288] como el impacto que genera la llegada de un partido recién formado y la desafección ciudadana hacia las organizaciones partidarias tradicionales, lo cual modifica el sistema de partidos mexicano.

Ahora bien, aunque resulte complejo aprehender el cambio en un presente en plena transformación, uno de los resultados indudables de estos comicios ha sido el fortalecimiento de la democracia mexicana in totto, ya que se ha producido una alternancia tanto partidaria como ideológica, que abre una nueva frontera a la política en México y —ipor qué no! - el tránsito hacia un sistema enteramente poliárquico (Dahl, 1989).

Estos elementos obligan a dar cuenta de la complejidad de la trama electoral de 2018. Sin embargo, en general, los estudios sobre elecciones tienden a ofrecer respuestas descriptivas o coyunturales, sin dar cuenta del entramado histórico político en el que se configuran. Para tal fin, se auscultará el diseño, mecánica y dinámica de la competencia electoral en México, desde su apertura a la competencia plural y democrática a finales del

${ }^{1}$ Formada por Morena, el Partido del Trabajo (PT), ambos de tendencia de izquierda progresista, y por el Partido Encuentro Social (PES), de tendencia conservadora e influencia cristiana. 
siglo; se abordará el surgimiento de Morena como nueva fuerza partidaria; y se analizarán las vicisitudes de la campaña y los resultados electorales. De esta manera, se ofrece una mirada compleja, a partir de una aproximación metodológica de tipo histórico-político, en la que el triunfo de AMLO-Morena pueda ser dimensionado adecuadamente.

\section{Radiografía del sistema de partidos en México}

Giovanni Sartori (2000) define al Partido Revolucionario Institucional (PRI) como un partido hegemónico-pragmático. Esta definición puede dar la apariencia, pero no la sustancia, de que la política es competitiva. No permite el enfrentamiento abierto ni el disenso efectivo y tampoco se aproxima a la oligarquía competitiva de Dahl (1989). Los partidos de «fuera» —afirma Sartori- no pueden convertirse jamás en partidos de «dentro» y serían una oposición tolerada. En México no hubo un partido totalitario, pero sí un partido hegemónico autoritario. El partido hegemónico sabe más y escucha más. No obstante, no existe ningún vínculo entre las exigencias y su satisfacción. Cualquiera que sea la información, el partido hegemónico puede imponer su voluntad.

La historia política de México contrasta con las abruptas transformaciones de régimen que sufrieron algunos países latinoamericanos en las décadas de 1960 a 1980 (Levitsky y Ziblatt, 2018). México experimentó una estabilidad política y una continuidad alrededor del PRI (Garrido, 1991), el cual había limitado a la representación simbólica el papel político de las fuerzas armadas en ciertos cargos públicos establecidos (Sánchez, 1988; Middlebrook, 1988). Pese a la institucionalización del Partido Nacional Revolucionario (PNR) antecedente del PRI-, en algunos momentos hubo ciertos liderazgos de oposición que desestabilizaron a la «familia revolucionaria»: Juan A. Almazán (1940), Ezequiel Padilla (1946) y Miguel H. Guzmán (1952). ${ }^{2}$ Sin embargo, entre 1952 y 1988, los desafíos al candidato presidencial del PRI fueron mínimos.

La ruptura significativa al interior del PRI se daría recién con la candidatura de Cuauhtémoc Cárdenas, ${ }^{3}$ sin embargo, en estas elecciones de

\footnotetext{
${ }^{2}$ Opositores al candidato oficial del PRI que, si bien en las campañas hicieron un ruido significativo, sus resultados oficiales fueron magros.

${ }^{3}$ Hijo del presidente Lázaro Cárdenas del Río (1934-1940), quien realizó la expropiación petrolera de 1938.
} 
julio de 1988 resultó ganador el priísta Carlos Salinas de Gortari, a pesar de los fuertes cuestionamientos a la legalidad y legitimidad del proceso, tildado incluso de fraude electoral (Aguayo, 2007, p. 118; Aitken, 2008, p. 420). Dicha elección significó la escisión más trascendental del PRI, pues políticos profesionales encabezados por Cuauhtémoc Cárdenas, Porfirio Muñoz Ledo, ${ }^{4}$ Andrés Manuel López Obrador, entre otros, fundaron el Partido de la Revolución Democrática, el cual acogió en sus filas a múltiples movimientos sociales (Combes, 2011). Además, esto coadyuvó a profundizar la alternancia subnacional, ya que entre 1989 y 1991 solo dos entidades federativas fueron gobernadas por el Partido Acción Nacional (PAN) (Langston, 2008; Modoux, 2006).

En 1994 nuevamente ganó el PRI con Ernesto Zedillo Ponce de León. La novedad en esta coyuntura fue la victoria electoral del PRD en 1997, tanto en el Distrito Federal como en el Congreso Federal, donde el PRI perdió la mayoría simple. Dichas transformaciones permitieron el tránsito hacia un sistema multipartidista (PRI, PAN y PRD), capaz de introducir en el sistema la alternancia pacífica en el poder (Alcántara, 2000). La tendencia hacia un sistema plural se mantuvo en las elecciones de 2000, en las que el PRI, por primera vez en su historia, perdió la Presidencia frente a un candidato de extracción panista, Vicente Fox Quesada.

En julio de 2006 se dio la elección presidencial más competitiva y polémica en la historia reciente de México, con una diferencia porcentual según los datos del INE entre el candidato del PAN, Felipe Calderón (35,89\%), y el candidato del PRD, AMLO (35,53\%), de solo 0,56\%, lo que generó una amplia polarización social y cuestionamientos a la legitimidad del ganador y al proceso electoral (IFE, 2006). Como consecuencia, Calderón llegó a la Presidencia con una escasa legitimidad y, en contrapartida, AMLO irrumpió en el centro de la escena pública al ocupar el eje vial más importantes del Distrito Federal y autodenominarse como «presidente legítimo».

En las elecciones presidenciales de 2012 Enrique Peña Nieto (PRI) logró triunfar frente a Josefina Vázquez Mota (PAN) y AMLO (coalición PRD-PTMovimiento Ciudadano), sin embargo, en 2018 AMLO encaró la contienda presidencial con un nuevo partido: Morena.

${ }^{4}$ Exmiembro del PRI — hasta 1987—, y ex secretario de Estado. Ha tenido múltiples cargos en la función pública. 


\section{La génesis del Morena como partido nuevo}

Un signo distintivo de Latinoamérica es el cambio político y la proliferación constante de nuevos partidos, siendo muy pocos los que consiguen establecerse como baluartes del sistema de partidos $y$, muchos menos, configurarse en opciones exitosas electoralmente (Kestler, Krause y Lucca, 2017).

Para comprender a Morena, en tanto nuevo partido, nos valdremos de un supuesto básico: los partidos políticos son una institución en sentido amplio que se organiza en pos de obtener, conservar y acrecentar el poder político representativo en disputa (Abal, 2002). Sin embargo, un partido, en tanto institución, puede ser pensado — simplificadamente- de tres maneras: el viejo institucionalismo puso el acento en las reglas formales del juego político; el nuevo institucionalismo de la acción racional puso su ahínco en el rol de los agentes de lo político; y el neoinstitucionalismo sociohistórico se ha focalizado en la transformación social de los «patrones de significación que guían la acción humana» (Hall y Taylor, 2003, p. 209). Tomando estas perspectivas como guía, se señalan tres opciones teóricas para pensar la formación de nuevos partidos y así reconocer el derrotero de Morena: la que coloca el acento en condiciones institucionales; la que pone el énfasis en la dinámica de los agentes; o bien aquella que recupera la importancia de los cambios socioestructurales (Krause, Kestler y Lucca, 2013).

Dentro de la mirada enfocada en las condiciones institucionales, algunos autores se enfocaron en el sistema electoral (Rae, Hanby y Loosemore 1971; Harmel y Robertson, 1985, p. 405). Señalan genéricamente que un sistema proporcional incentivaría la formulación de nuevas siglas partidarias, en tanto que un sistema mayoritario lo inhibiría. En el caso mexicano prima un sistema electoral mixto, en el que el legislativo se integra mediante distritos de mayoría relativa y otros de representación proporcional. Desde su inscripción como partido el 9 de julio de 2014, Morena tuvo la oportunidad de participar en la disputa electoral legislativa de 2015 y, posteriormente, en las contiendas estaduales de 2016 y 2017, las cuales fueron un acicate para su crecimiento organizativo y su incursión electoral de cara a la contienda presidencial de 2018.

Dentro de la perspectiva institucional formal, otros autores ponen el acento en las características del distrito electoral (Cox, 1997, p. 203; Taagepera 
y Shugart, 1989, p. 112), al señalar que un distrito de gran magnitud favorece la incorporación de nuevos contendientes partidarios, en contraste, las circunscripciones uninominales lo desalientan. En el caso mexicano, distritos de gran magnitud como el entonces D. F. y Veracruz sirvieron de plataformas para la génesis de Morena y su estreno electoral exitoso en 2015 (Navarrete y Espinoza, 2017).

Otros autores ponen el énfasis en las barreras para la inscripción de nuevas siglas partidarias para los casos latinoamericanos (Hug, 2000; Mustapic, 2013, p. 207). Señalan que unos requisitos institucionales más laxos para formular legalmente siglas partidarias, o incluso para permitirles su supervivencia electoral o financiera, favorecen la incursión de nuevos contendientes en el sistema de partidos. En el caso mexicano, aunque la barrera electoral o umbral de exclusión es comparativamente baja (3\%), el artículo 11 de la Ley General de Partidos Políticos ha servido históricamente como un desincentivo institucional para la inscripción de nuevas organizaciones, puesto que cualquier sigla novel con vocación de disputar la Presidencia como era el caso de Morena y AMLO — debería tomar esa decisión cinco años antes, lo cual es un plazo lejano para el cálculo electoral o su supervivencia organizativa y financiera.

Por último, existen otras explicaciones institucionales de tipo formal que ponen el énfasis en la forma de gobierno — presidencial versus parlamentaria - o la distribución territorial del poder - gobierno unitario y centralizado versus federal y descentralizado-. En cuanto a la primera cuestión, para Latinoamérica se plantea que el presidencialismo facilita el ingreso de figuras a título individual —outsiders - con capacidad para formar partidos ad hoc para competir electoralmente (Hauss y Rayside, 1978, p. 44; Mainwaring, Gervasoni y Nájera, 2010, p. 16). En cuanto al segundo aspecto, se apunta a que mayores niveles de distribución territorial del poder abren mayores espacios para la incorporación de nuevos actores partidarios (Lijphart, 2000, p. 185; Lucardie, 2000, p. 180).

Contrariamente a lo que señalaría la literatura y la experiencia, en el caso mexicano fue el PRI el que incorporó a un outsider del partido como candidato presidencial, José Antonio Meade Kuribreña. En contraste, en el caso de Morena, aunque AMLO se presentó como un outsider de los partidos establecidos, gracias a su prédica movimientista y social, él es plenamente un insider de la política mexicana, habida cuenta su previa adhesión tanto al PRI 
(1970-1988) como al PRD (1988-2012), además, la estructura organizativa de Morena no difiere sustantivamente de los demás partidos tradicionales.

En cuanto a la distribución territorial del poder, aunque sería de esperar que Morena tuviera un modelo genético por difusión (Panebianco, 1990), habida cuenta la histórica concentración de poder del PRI, su característica genética principal es la de una penetración territorial anclada fundamentalmente en la Ciudad de México, de forma analógica al proceso de surgimiento de nuevos partidos desafiantes latinoamericanos en San Pablo, Brasil, con el Partido de los Trabajadores, o en Montevideo, Uruguay, con el Frente Amplio.

Dentro de la mirada teórica que pone el acento en los actores y su agencia al momento de formar nuevos partidos, muchos puntualizaron el rol nodal de los líderes (Panebianco, 1990; Aldrich, 1995; entre otros). En los estudios partidarios latinoamericanos el liderazgo ha sido una explicación preponderante y recurrente, al ponderar el rol del caudillismo electoral (Baquero, 2000, pp. 72-73) o la proliferación de «políticos sin partidos» (Cavarozzi y Casullo, 2002, p. 14), incluso el extenso anaquel de abordajes en torno a un liderazgo populista (Freidenberg, 2007). Esta perspectiva enfocada en el líder es una de las más productivas en México dentro de la Ciencia Política, el debate público y la militancia de Morena, puesto que consideran que AMLO tuvo una centralidad - y centralización — inusitada en el proceso de formación de esta organización política en múltiples momentos históricos: primero, al destacarse como jefe de Gobierno del D. F. por encima de su adscripción al PRD (2000-2005); segundo, por la gran activación de masas que generó el intento de desafuero en $2005 ;{ }^{5}$ en tercer lugar, por las movilizaciones en el D. F. durante la impugnación del fraude de 2006 en contra de Felipe Calderón, que a posteriori le ayudaron a capitalizar sus bases. Por ende, al lopezobradorismo, en tanto movimiento, solo le restaba la obtención formal del registro electoral para autopercibirse como partido (Bolívar, 2014).

Dentro de esta extensa mirada en torno a la importancia de los líderes para explicar la formulación de nuevos partidos, algunos han puesto el énfasis en el cálculo estratégico de costo-beneficios que las dirigencias políticas realizan para saber si conviene ingresar en la disputa electoral con

${ }^{5}$ El gobierno de Vicente Fox (2000-2006) propuso quitarle el fuero político a AMLO en 2005 para que pudiera ser juzgado por un tribunal. 
una nueva sigla, mantenerse dentro de un partido o bien migrar hacia otra organización partidaria (Aldrich, 1995, p. 36; Mair, 1999, p. 8; Tavits, 2008; Lago y Martínez, 2011, p. 8). En el caso de Morena, a pesar del encono entre el lopezobradorismo y la dirigencia del PRD, durante la primera década del siglo Xxı resulta llamativo que el vínculo AMLO-PRD no se disolviera antes. Además del desincentivo institucional, es un hecho incontrastable que el lopezobradorismo no contaba con los recursos materiales y organizativos como para desprenderse rápidamente del PRD. A pesar de una historia de múltiples encontronazos entre AMLO y el PRD, la firma en 2012 del Pacto por México entre el presidente Peña Nieto y líderes de distintos partidos políticos como Gustavo Madero (PAN) y Jesús Zambrano (PRD) fue el catalizador para oficializar la escisión del lopezobradorismo del PRD y volver partido a Morena. ${ }^{6}$

Dentro de la perspectiva que hace foco en los actores, otros ponen el acento en las organizaciones auspiciantes de los nuevos partidos políticos, la transformación de los movimientos sociales en nuevas organizaciones partidarias, el ingreso de nuevas élites y bases sociales en la formulación de una nueva sigla partidaria, entre otros aspectos (Hauss y Rayside, 1978, p. 43; Ignazi, 1996, p. 555; Perkins, 1996, p. 368; Lucardie, 2000, pp. 176179). En México, gran parte de la politología y el debate público señalan a AMLO como un líder social con ribetes populares y a Morena como un movimiento social electoral que luego devino en partido. Como se señaló, AMLO ha sido siempre un líder netamente político, ya que su foco nunca se corrió de la disputa electoral o de las instituciones partidarias, con lo cual sería inconducente considerarlo un líder de origen social que encauzó un movimiento social en un partido, como es el caso de Evo Morales en Bolivia o de Lula da Silva en Brasil. En cuanto a si Morena proviene enteramente de un movimiento social, cabe señalar que al momento de su inscripción como partido converge, por un lado, un gran caudal de lopezobradoristas que sustentaron este movimiento electoral en los agravios del desafuero (2005), el fraude electoral (2006) y la violencia y corrupción política; por el otro, un gran acervo de líderes y dirigentes provenientes del PRD y partidos de izquierda como el PT a lo largo y ancho del territorio. Asimismo, resultaría

\footnotetext{
${ }^{6}$ Paradójicamente, este momento de mayor distancia ideológica entre AMLO-Morena con los partidos establecidos convive con un creciente pragmatismo estratégico de AMLO, que recupera principios clásicos del PRI — como el nacionalismo revolucionario-, realiza una moderación discursiva — con consignas como la de una «república amorosa»- y esboza alianzas electorales heterogéneas (Monsiváis, 2018, pp. 17 y 21).
} 
difícil considerar plenamente al movimiento electoral, gestado entre 2005 y 2014, como un movimiento social, ya que su espacio preferente de acción fue el institucional y su relación con el poder — aunque conflictiva - se anclaba en la pretensión de reforma y reemplazo de la elite gobernante, lo cual lo distancia de una definición clásica de movimiento social (Marti i Puig y Rovira i Sancho, 2017, pp. 281-282).

Un párrafo aparte dentro de esta literatura sobre la formación de nuevos partidos por la dinámica de los actores lo merecen las formulaciones que vinculan el origen en relación con los partidos establecidos, ya sea por su fraccionamiento interno, el transfuguismo, la falta de cohesión, la formación de coaliciones, entre otros. Sin embargo, este tipo de perspectivas necesariamente deben distinguir cuál es la «novedad» de un «partido nuevo» heredero de partidos tradicionales (Mair, 1999, p. 216; Sikk, 2012; Barnea y Rahat, 2011, pp. 305 y 309). En el caso mexicano, aunque esta perspectiva parece ofrecer una respuesta para explicar la incubación de Morena dentro del PRD, no debe opacar la experiencia del lopezobradorismo en tanto movimiento electoral en el que se incorporaron múltiples dirigentes y participantes por fuera del PRD y la izquierda partidaria.

Una última vía analítica para pensar la formación partidaria es aquella que pone el énfasis en cambios socio-estructurales. Entre los desarrollos más extendidos en esta clave están los que se articulan en torno a la noción de clivaje, en tanto fracturas socialmente relevantes que se tornan políticamente sustantivas en la disputa política y la génesis partidaria, luego de la revolución industrial y la configuración del Estado-Nación (Lipsety Rokkan, 1967). Empero, especialmente a partir de las décadas de 1960 y 1970, múltiples autores formularon la incorporación de nuevas transformaciones sociohistóricas que derivaron en la aparición de nuevos grupos sociales y orientaciones normativas —value change-, dando lugar a nuevos partidos (Inglehart, 1977; Ignazi, 1996, p. 556; Kitschelt, 1994; Hug, 2000; van Biezen, 2003; Sikk, 2012). En el caso latinoamericano, la hipótesis de la génesis partidaria a partir de clivajes se ha abordado en las propuestas de Robert Dix (1992), acerca del desfase entre los procesos históricos propuestos por Lipset y Rokkan; la singularidad de los cismas o revoluciones latinoamericanas donde se forjan los clivajes y partidos latinoamericanos —oligárquica, nacional-popular y democráticasegún Alfredo Ramos Jiménez (2001, p. 88); o múltiples estudios de casos que distinguen clivajes ad hoc a cada caso estudiado. 
Paralelamente, otra gran formulación en torno a la génesis partidaria por cambios socio-estructurales, puso el acento en las crisis y en las coyunturas críticas. Sobre las primeras se formuló un modelo explicativo que vinculaba el surgimiento de nuevos partidos con las crisis inherentes al proceso de modernización —legitimidad, integración o de participación(LaPalombara y Weiner, 1966, p. 14). En cuanto a las segundas, se planteó que en estos «momentos de verdad» se produce la fusión o escisión de partidos preestablecidos, el surgimiento de nuevos liderazgos y, por ende, la formación de nuevos partidos (Janda, 1980). Para Latinoamérica, uno de los trabajos seminales sobre coyunturas críticas y nuevas fuerzas políticas es el de Ruth Collier y David Collier (2002).

Al pensar el origen de Morena, su génesis se ancla en su animadversión frente a los partidos establecidos, proponiendo un claro clivaje en términos de continuidad-cambio o establishment-anti-establishment. Si a esto le añadimos la crisis de legitimidad que vivía el sistema político por el hartazgo frente a la corrupción y los niveles de violencia, es posible comprender cómo se favoreció Morena de una estructura de oportunidades histórica para plantear una alternativa electoralmente creíble para los comicios de 2018.

\section{[296] 3. La campaña electoral de 2018 y el camino de Morena al éxito electoral}

A pesar de que AMLO siempre fue puntero en las encuestas, las campañas desempeñan un papel determinante, ya que no solo ofician en la formación de las preferencias del electorado indeciso, sino que influyen también en las estrategias de campaña de los candidatos. En este sentido, tanto el PRI como el PAN, sabiendo de su desventaja inicial, buscaron capitalizar los resquicios para mejorar su desempeño.

En noviembre de 2016 la encuestadora BGC-Excélsior ${ }^{7}$ consideraba que no existía ningún partido puntero para la contienda presidencial de 2018, por lo tanto, se preveía una contienda extremadamente cerrada: el PAN con una intención de voto de 30\%, el PRI con 28\% y Morena con 20\%. Dicha encuesta preguntó quién debería ser el candidato de cada partido: por el PRI, el mejor evaluado fue Miguel Ángel Osorio, secretario de Gobernación, con

\footnotetext{
${ }^{7}$ Para efectos del presente artículo, se enfocará en las cuatro mayores encuestadoras del país: Encuesta Reforma; BGC-Excélsior; Parametría; Consulta Mitofsky; y Pew Research Center, con sede en Washington.
} 
22\% y José Antonio Meade con 1\%; por el PAN, Margarita Zavala, esposa del expresidente Calderón, presentaba 27\% de aprobación, en tanto Ricardo Anaya contaba con $10 \%$; por Morena, AMLO dominaba con $34 \%$ y Miguel Mancera con 13\% (Beltrán y Cruz, s. f.).

BGC-Excélsior planteó ciertos «careos» entre los posibles candidatos. El PAN ganaría - por un corto margen — si postulaba a Zavala y en cualquier otro escenario caería al tercer lugar y AMLO ganaría con + 4 la Presidencia. No obstante, preveía que, aunque Zavala fuera la candidata, si el PAN formaba una coalición con el PRD Zavala caería al tercer lugar. Ambos partidos se debilitaban más que potenciarse. Por otro lado, si Morena se aliaba con el PRD no obtendría ganancia electoral alguna, como sí se lo ofrecía coaligarse con el PT (Beltrán y Cruz, s. f.).

A pesar de los pronósticos de BGC-Excélsior, el PAN decidió formar una coalición con el PRD y elegir a Anaya como candidato. Aunque esta coalición había funcionado en estados como Quintana Roo, en este caso fue contraproducente, porque desdibujó ideológicamente a su electorado y generó un cisma al interior del PAN, tanto por la salida de Zavala como por los cuestionamientos que hicieron dirigentes del PAN como Gustavo Madero, expresidente del partido.

Por su parte, la coalición México al Frente, ${ }^{8}$ liderada por el PRI, nominó como candidato «ciudadano» a José Antonio Meade, un outsider con escasa conexión con el electorado del partido. Su candidatura se veía signada tanto por el peso de $69 \%$ de mexicanos que sentían un deterioro en la situación económica (Beltrán, 2018, julio 2), como por la caída estrepitosa de la imagen del presidente Peña Nieto, quien pasó de 61\% de aprobación en 2011, a 28\% en 2017 (Vice y Chwe, 2017).

En lo que respecta a Morena, profundizó su alejamiento del PRD y formó la coalición Juntos Haremos Historia con el PT y el PES. Aunque Morena se autodefine de izquierda y progresista, el PES tiene una postura conservadora religiosa, lo cual evidencia una coalición anómala en términos ideológicos.

Entonces, considerando la proyección hecha por Ulises Beltrán y Alejandro Cruz (s. f.) y las decisiones del PRI y PAN, es posible discernir que

${ }^{8}$ Junto con el Partido Verde Ecologista de México y el Partido Nueva Alianza. 
el magro desempeño electoral de ambos partidos se explicaría por escoger el peor de los escenarios posible, tanto en la nominación de candidatos como en la conformación de sus coaliciones. En contraste, Morena eligió las mejores opciones proyectadas por la encuesta BGC-Excélsior. Las coaliciones para la elección presidencial 2018 dieron muestras de un extremado pragmatismo, en el que se privilegió la obtención de votos más que la congruencia ideológica. ${ }^{9}$

¿Qué sucedió durante la campaña electoral y por qué la brecha se ensanchó tanto? La Encuesta Reforma del mes de febrero le dio a Anaya 32\% de la intención de voto, 10 puntos por debajo de AMLO, con 42\% (Becerra, 15 de febrero de 2018). No obstante, Lorena Becerra (18 de abril de 2018) reveló que el margen de ventaja se había duplicado a favor de AMLO (22\%). Para explicar la torsión irremontable entre ambas encuestas, hay que tener en cuenta las denuncias de corrupción que aparecen contra Anaya, acusándolo «de triangular fondos en paraísos fiscales por varios países y lavar dinero para la compra de una nave industrial» (Olivares, 2018, febrero 20).

Aunque AMLO casi duplicaba la intención de voto de Anaya - $48 \%$ y $26 \%$, respectivamente-, su coordinador de campaña, el excanciller y académico Jorge G. Castañeda, afirmó que si Anaya se tornaba competitivo

[298] los «votantes blandos» de AMLO se irían acercando. Sin embargo, la estrategia de Anaya fue errónea, ya que se esmeró en atacar al PRI sin darse cuenta de tres cosas: a) que no estaba en 2000, cuando ese discurso funcionó; b) que el PAN no representaba ninguna renovación sino parte del establishment vinculado a la corrupción que denunciaba; y c) que el enemigo a vencer era fundamentalmente AMLO.

En cuanto al desempeño de campaña de AMLO, su estrategia puede dividirse en tres aspectos: la estrategia territorial, mediática y tras bambalinas. En cuanto a la primera, AMLO llevaba años recorriendo el país, teniendo una amplia presencia tanto en el México rural como urbano; en segundo lugar, AMLO evitó ser frontal ante los ataques mediáticos de sus contendientes, transmitiendo tranquilidad, incluso frente a la campaña sucia que lo tildaba de amenaza castrochavista; en tercer lugar, AMLO emprendió una extensa

\footnotetext{
${ }^{9}$ La encuestadora Parametría (Abundis, 2018, julio 19) analizó el momento en el que los votantes definieron su preferencia electoral. En este sentido, Anaya y AMLO lograron seducir con éxito a su electorado durante la campaña - $40 \%$ y $38 \%$ respectivamente-, en contraste con el magro poder de convocatoria de Meade. Incluso, $40 \%$ de los votantes de Morena decidió su voto cuando supo que AMLO sería el candidato.
} 
agenda de reuniones cerradas con diversos actores políticos, sociales, religiosos y empresariales, por ejemplo, se realizaron acercamientos, a través del empresario Alfonso Romo y la expanista Tatiana Clouthier, con el Consejo Coordinador Empresarial, un grupo abiertamente hostil a AMLO en 2006.

\section{La elección, sus resultados e interpretaciones}

A lo largo de su gobierno, Peña Nieto tuvo como horizonte de gestión «mantener la estabilidad macroeconómica» (Borovkov, 2018, pp. 9-11). Sin embargo, la percepción de que la situación económica empeoró en su gobierno rondaba 69\% (Beltrán, 2018, julio 2). La ciudadanía mexicana mostraba así claros signos de agotamiento por los altos niveles de pobreza, corrupción, violencia, impunidad y desigualdad extrema (Esquivel, 2015).

Un supuesto es que este «descontento» del rumbo económico es una «metástasis» de otras dos situaciones: por un lado, el impacto de la inseguridad cotidiana por la «guerra contra y entre el narco», configurando uno de los periodos más violentos del México contemporáneo (Inegi, s. f.) $i^{10}$ por el otro, de acuerdo con la encuesta de Pew Research Center (Vice y Chwe, 2017, p. 13), la corrupción es considerada por $84 \%$ de los encuestados como uno de los principales problemas del país. Combatir al crimen y a la corrupción fueron justamente las banderas que AMLO eligió como eje de su campaña, lo cual, sumado al descrédito y encono de la administración de Peña Nieto, se convirtieron en la plataforma para ganar la Presidencia en su tercer intento.

AMLO obtuvo la victoria a la Presidencia con $53 \%$ de los votos, frente a 22\% que consiguió Anaya y $16 \%$ de Meade (INE, 2018). Frente a esta arrolladora victoria, cabe preguntarse iqué hizo AMLO en esta ocasión a diferencia de sus campañas anteriores para obtener la victoria? Una respuesta posible la ofrece el desempeño y enraizamiento territorial del voto a través de las regiones. Si se observa el mapa electoral antes de 2018, analizando la afiliación partidaria de cada gobernador y el voto ciudadano en las últimas tres elecciones presidenciales, resulta evidente que México se encontraba fragmentado electoralmente: en 2006 entre el sur-centro (AMLO-PRD) y norte (Calderón-PAN); en el 2012 entre sur (AMLO-PRD)

\footnotetext{
${ }^{10}$ Desde el sexenio de Calderón y durante el de Peña Nieto, las tasas de homicidio dolosos han fluctuado desde 2009, alrededor de 20 mil homicidios por año (Inegi, s. f.).
} 
y norte-centro (EPN-PRI). Es decir, el norte y la península de Yucatán han sido propensas a fluctuar entre el PAN y el PRI, el PRD no ha tenido ningún gobernador en los estados fronterizos, solo Zacatecas es un enclave del PRD. En el sur-centro se tiende a votar por el PRD o PRI. Es por lo tanto visible que tanto el PAN era incapaz de penetrar en el sur-centro, como AMLO-PRD en el norte.

Para comprender con mayor detalle la metamorfosis del enraizamiento territorial del voto partidario, es posible analizar los comportamientos electorales en las distintas entidades. Para ello, cabe tomar en cuenta que el Instituto Nacional Electoral divide al país en cinco circunscripciones electorales con igual cantidad de población: la Circunscripción \#1 en el noroeste, que incluye los Estados de Baja California, Baja California Sur, Chihuahua, Durango, Jalisco, Nayarit, Sinaloa y Sonora; la Circunscripción \#2 o noreste, conformada por Aguascalientes, Coahuila, Guanajuato, Nuevo León, Queretáro, San Luis Potosí, Tamaulipas y Zacatecas; la Circunscripción \#3 o sureste integrada por Campeche, Chiapas, Oaxaca, Quintana Roo, Tabasco, Veracruz y Yucatán; la Circunscripción \#4 o centro-sur, compuesta por Ciudad de México, Guerrero, Morelos, Puebla y Tlaxcala; y la Circunscripción \#5 o centro, que aúna Colima, Hidalgo, México y Michoacán.

La gráfica 1 permite desglosar la tendencia del voto en las elecciones de 2006 (IFE, 2006), 2012 (INE, 2012) y 2018 (INE, 2018). En las elecciones de 2006 y 2012 los votantes de AMLO (amarillo) en la C\#1 y C\#2 se mantienen estables $-24 \%$ y $20 \%$, respectivamente-, lo cual permite advertir la existencia de un voto duro sumamente leal, pero, en contrapartida, un techo para seducir a otros votantes.

Al considerar solo la C\#1 en 2006 se observa que es donde mayor apoyo recibió Calderón — 48\%, azul marino- para ejercer contrapesos territoriales frente a otras circunscripciones, por ejemplo, la C\#4, donde AMLO obtuvo 50\%. Es verosímil deducir que el electorado de la C\#1 que apoyó a Calderón en 2006 decidió castigarlo en 2012 —posiblemente frente a la ola de inseguridad - votando por el PRI —Peña Nieto rojo- pero sin optar por AMLO, quien conservó su voto duro en ambas elecciones. En 2018, AMLO ganó en todas las circunscripciones, pero es en la C\#1 donde superó por $15 \%$ su techo histórico, penetrando por primera vez en el norte $-24 \%$ a $39 \%$ - 
Las elecciones de 2018 en México y el triunfo del Movimiento de Regeneración Nacional...

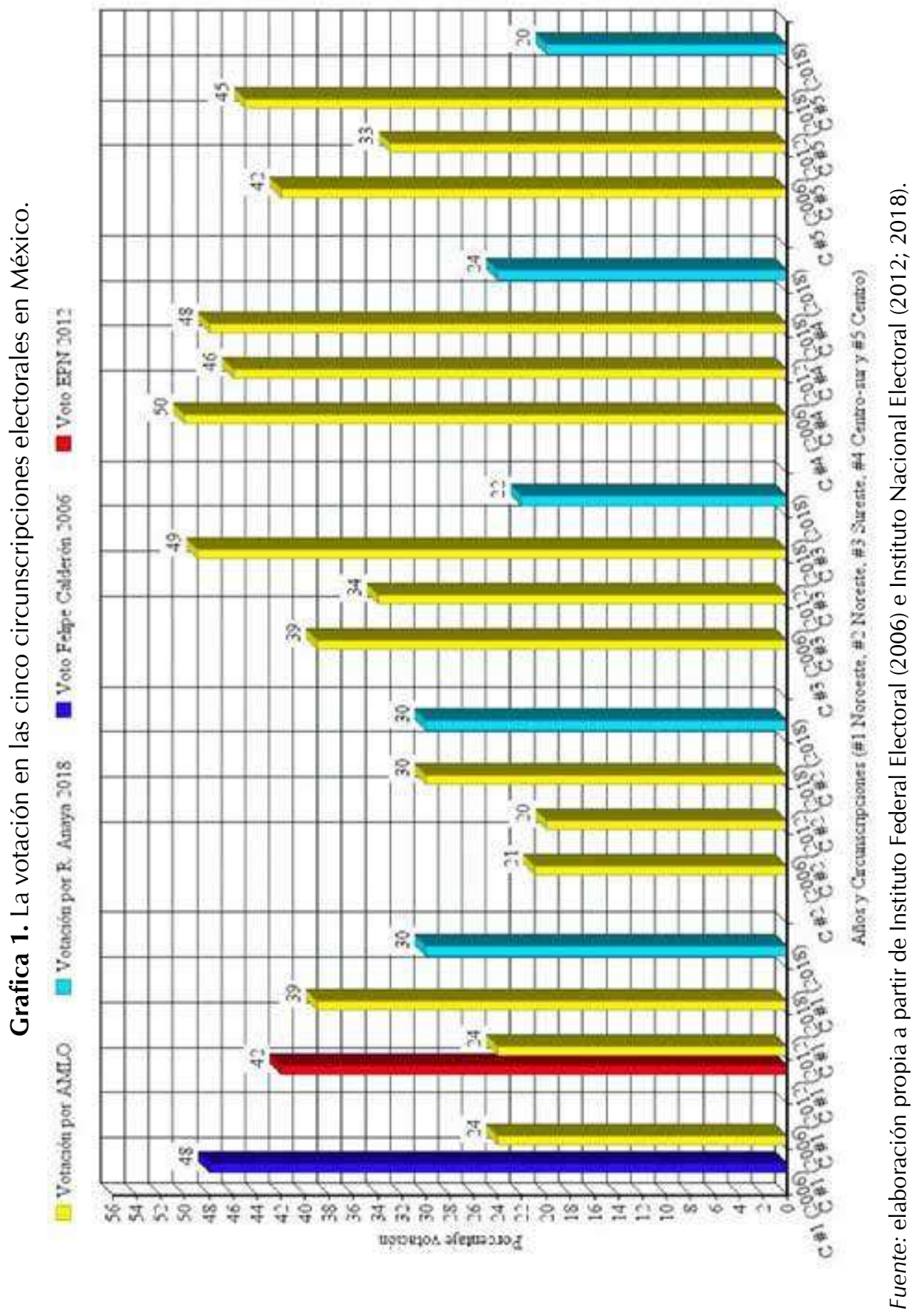


Si bien la C\#2 confirma que AMLO logró penetrar en el norte —creció $10 \%$ - también muestra que su desempeño fue el menos rotundo, siendo la única entidad en la que empató con Anaya. En las otras tres circunscripciones -C\#3, C\#4 y C\#5- se observa que en 2012 hubo un verdadero «efecto Peña Nieto» (Villamil, 2012), ya que el voto para AMLO tiene una caída significativa. Este efecto es flagrante en la C\#5, donde el exgobernador del Estado de México le quitó casi 10 puntos a AMLO en relación con 2006; sin embargo, al compararlo con 2018, AMLO logró recuperar su ventaja: en la C\#3 superó por $10 \%$ su voto histórico; en la C\#5 solo por 3\%; y en la C\#4 tiene un comportamiento constante, ya que el efecto Peña tuvo poca incidencia.

En síntesis, el análisis de las circunscripciones evidencia que para la elección de 2018 AMLO creció en todas las circunscripciones, siendo este equilibrio territorial el principal cambio frente a otras elecciones. En la C\#1 penetró por primera vez; en la C\#2 debilitó al PAN; en la C\#3 recuperó su credibilidad; en la C\#4 se estableció su bastión; y en la C\#5 fluctuó entre apoyarlo o darle la espalda. Quitando el difícilmente reproducible «efecto Peña Nieto» - en las C\#5 y C\#3-, en los comicios de 2018 se rompió la impenetrabilidad en las $\mathrm{C \#} 1$ y C\#2, y es posible pensar en estas regiones como swing states a la mexicana, es decir, son zonas con una volatilidad elevada del voto: apoyaron al PAN en 2006, al PRI en 2012 y a Morena en 2018.

Una posible interpretación de esta volatilidad electoral en las C\#1 y C\#2 puede deberse, por un lado, a las fuertes acusaciones de corrupción a los gobernadores de Chihuahua en la C\#1, y Coahuila y Tamaulipas de la C\#2; por el otro, que han sido los principales territorios de lucha del crimen organizado. Considerando la tasa de homicidio por entidades más violentas de México, según el Instituto Nacional de Estadística y Geografía (2018) ( $>3,5 \%$ de los homicidios totales) $7 / 10$ de los estados muestran presencia del crimen organizado y 5/7 hacen parte de las C\#1 y C\#2. De los estados con más de 25 homicidios por cada 100 mil habitantes (Inegi, s. f.) 4/5 pertenecen a las C\#1 y C\#2 —Chihuahua, Sinaloa, Baja California Norte y Tamaulipas-.

\section{Conclusiones. ¿Nace un nuevo sistema político en México?}

La estabilidad política en México a lo largo del tiempo, comparándola con los altibajos de sus pares latinoamericanos, es un dato llamativo; sin embargo, el último proceso electoral de 2018 muestra que el sistema de partidos que se conformó en México durante las tres últimas décadas 
claramente ha cambiado. Los tres grandes partidos históricos —PRI, PAN y PRD — han sido desplazados en todos los ámbitos — federal, legislativo y subnacional_- por un nuevo contendiente: Morena.

Si bien el PRI perdió las elecciones presidenciales de 2000 y 2006, nunca había quedado tan mermada su fortaleza en el Legislativo y en las entidades federativas, muchas de las cuales se habían mantenido como bastiones clave para su sobrevivencia. En un hecho sin precedentes, el PRI solo obtuvo 45/500 diputados federales y 14/128 senadores. Esta debacle del PRI también se expresa en el ámbito subnacional con las gubernaturas estatales. En 2012, cuando el PRI recuperó la Presidencia, obtuvo también 21/31 gubernaturas. En la elección de 2018, de 8 gubernaturas en disputa el PRI no obtuvo ninguna y perdió Yucatán y Jalisco. A la fecha, solamente 5 estados - Coahuila, Hidalgo, Colima, Estado de México y Campeche- no han sido gobernados por un partido diferente al PRI. Por tanto, los resultados de la última elección son los peores en toda la historia del PRI.

En contraste, el candidato de Morena fue el más votado en todos los estados de México, con la excepción de Guanajuato. Obtuvo 4 gubernaturas, incluyendo la Ciudad de México, y logró 252/500 curules en la Cámara de Diputados, a los que se pueden agregar 31 de su aliado el PT. En el Senado de la República obtuvo 59/128 escaños que conforman el hemiciclo, más 6 del PT. Esto hace que López Obrador disponga de un fuerte respaldo en el Poder Legislativo, lo cual permite vislumbrar un Poder Ejecutivo fuerte y con un amplio margen de maniobra para desarrollar su agenda, sin muchas restricciones o negociaciones complejas con los partidos de oposición.

Como se ha mostrado, tanto en los ámbitos federal como subnacional y legislativo, el PRI quedó muy mal parado en términos electorales y políticos. Con esto no se pretende decir que el PRI desaparecerá, pero el escenario que se le presenta es bastante complejo, ya que parece que su lugar será ocupado ahora por Morena como un partido catch all en el que conviven actores con ideologías de izquierda, centro y derecha, y miembros provenientes de diversos sectores de la sociedad, desde políticos tradicionales hasta académicos y empresarios, expriístas, expanistas y experredistas.

Morena se vislumbra como el PRI del siglo XXI, con la salvedad de que no es el PRI que tomaron tecnócratas adherentes al neoliberalismo a finales de la década de 1980, sino más bien un PRI similar al que defendía el 
intervencionismo estatal; además, Morena obtiene su legitimidad mediante un proceso democrático, a diferencia de la hegemonía del PRI, que desde finales de la década de 1960 perdió su legitimidad y se sostuvo en el poder a partir de elecciones cuestionadas, poco transparentes y fraudulentas.

Ahora habrá que esperar que las promesas de campaña se conviertan en verdaderas políticas gubernamentales y políticas públicas encauzadas a través de los canales institucionales adecuados para solucionar los problemas sociales que aquejan a México. Si esto sucede entonces sí se podrá hablar de una transformación política y no solo de un cambio en el sistema de partidos o la mera sustitución del PRI.

\section{Referencias bibliográficas}

1. Abal Medina, Juan Manuel. (2002). Elementos teóricos para el análisis contemporáneo de los partidos políticos: un reordenamiento del campo semántico. En: Cavarozzi, Marcelo y Abal Medina, Juan Manuel (comps.). El asedio a la política. Los partidos latinoamericanos tras la década del neoliberalismo (pp. 33-54). Rosario: Homo Sapiens.

2. Abundis, Francisco. (2018, julio 19). Identidades, candidatos, campañas y corrupción. Milenio. Recuperado de http://www.milenio.com/opinion/franciscoabundis/columna-francisco-abundis/identidades-candidatos-campanas-y-corrupcion

3. Aguayo, Sergio. (2007). Vuelta en U. México, D. F.: Taurus.

4. Alcántara, Manuel. (2000). Sistemas políticos de América Latina: Volumen segundo, México, los países del Caribe y América Central. Madrid: Tecnos.

5. Aldrich, John. (1995). Why Parties: The Origin and Transformation of Political Parties in America. Chicago: University of Chicago. https://doi.org/10.7208/ chicago/9780226012773.001.0001

6. Aitken, Rob. (2008). Carlos Salinas de Gortari. En: Fowler, Will. Gobernantes mexicanos. Tomo II 1911-2000. México, D. F.: FCE.

7. Baquero, Marcelo. (2000). A vulnerabilidade dos partidos políticos e a crise da democracia na América Latina. Porto Alegre: UFRGS.

8. Barnea, Shlomit y Rahat, Gideon. (2011). "Out with the Old, in with the 'New'": What Constitutes a New Party? Party Politics, 17 (3), pp. 303-320. https://doi. org/10.1177/1354068810369148

9. Becerra, Lorena. (15 de febrero de 2018). Lidera AMLO; sube Anaya y Meade se rezaga [Mensaje en un blog]. Encuestas Grupo Reforma. Recuperado de https:// gruporeforma-blogs.com/encuestas/?p $=7668$

10. Becerra, Lorena. (18 de abril de 2018). Consolida AMLO ventaja [Mensaje en un blog]. Encuestas Grupo Reforma. Recuperado de https:/gruporeforma-blogs.com/ encuestas/? $p=7735$ 
Las elecciones de 2018 en México y el triunfo del Movimiento de Regeneración Nacional...

11. Beltrán, Ulises. (2018, julio 2). Apoyo abrumador a López Obrador entre los ingresos más bajos de ingreso. Excélsior. Recuperado de https://www.excelsior.com. $\mathrm{mx} /$ nacional/apoyo-abrumador-a-amlo-los-mas-pobres-le-dieron-el-triunfo/1249582

12. Beltrán, Ulises y Cruz, Alejandro. (s. f.). Encuesta BGC-Excélsior. Van empatados rumbo a 2018; las preferencias se centran en PRI y PAN. BCC. Recuperado de http://bgc.com.mx/encuesta-bgc-excelsior/501-van-empatados-rumbo-a-2018-laspreferencias-se-centran-en-pri-y-pan

13. Bolívar Meza, Rosendo. (2014). MORENA: el partido del lopezobradorismo. Polis, 10 (2), pp. 71-103.

14. Borovkov, Anatoly. (2018). México ante la opción electoral, Iberoamerica, 1, pp. 5-23.

15. Cavarozzi, Marcelo y Casullo, Esperanza. (2002). Los partidos políticos en América Latina hoy: ¿Consolidación o crisis? En: Cavarozzi, Marcelo y Abal Medina, Juan Manuel (comps.). El asedio a la política. Los partidos latinoamericanos en la era neoliberal (pp. 9-30). Rosario: Homo Sapiens.

16. Collier, Ruth y Collier, David. (2002). Shaping the Political Arena: Critical Junctures, the Labor Movement, and Regime Dynamics in Latin America. Notre Dame: University of Notre Dame.

17. Combes, Hélène. (2011). Faire Parti. Trajectoires de gauche au Mexique, Paris: Karthala.

18. Cox, Gary. (1997). Making Votes Count: Strategic Coordination in the World's Electoral Systems. Cambridge: Cambridge University. https://doi.org/10.1017/ CBO9781139174954

19. Dahl, Robert. (1989). La poliarquía. Participación y oposición. Madrid: Tecnos.

20. Dix, Robert. (1992). Democratization and the Institutionalization of Latin American Political Parties, Comparative Political Studies, 24 (4), pp. 488-511. https:// doi.org/10.1177/0010414092024004004

21. Esquivel, Gerardo. (2015). Desigualdad extrema en México. México, D. F.: Oxfam.

22. Freidenberg, Flavia. (2007). La tentación populista: una vía al poder en América Latina. Madrid: Síntesis.

23. Garrido, Luis Javier. (1991). El Partido de la Revolución Institucionalizada. Medio siglo de poder político en México: La formación del nuevo estado (19281945). México, D. F.: Siglo xxı.

24. Hall, Peter y Taylor, Richard. (2003). The Three Versions of NeoInstitutionalism. Lua Nova: revista de cultura e política, 58, pp. 193-223. https://doi. org/10.1590/S0102-64452003000100010

25. Harmel, Robert y Robertson, John. (1985). Formation and Success of New Parties: A Cross-National Analysis. International Political Science Review, 6 (4), pp. 501-523. https://doi.org/10.1177/019251218500600408 
26. Hauss, Charles y Rayside, David. (1978). The Development of New Parties in Western Democracies Since 1945. En: Cooper, Josep y Maisel, Louis (eds.). Political Parties: Development and Decay (pp. 31-57). Beverly Hills: Sage.

27. Hug, Simon. (2000). Studying the Electoral Success of New Political Parties: A Methodological Note. Party Politics, 6 (2), pp. 187-197. https://doi. org/10.1177/1354068800006002004

28. Ignazi, Piero. (1996). The Crisis of Parties and the Rise of New Political Parties. Party Politics, 2 (4), pp. 549-566. https://doi.org/10.1177/1354068896002004007

29. Inglehart, Ronald. (1977). The Silent Revolution. Changing Values and Political Styles among Western Publics. Princeton: Princeton University.

30. Instituto Federal Electoral (IFE). (2006). Elección de presidente de los Estados Unidos Mexicanos. Cómputos distritales de las elecciones federales de 2006. Resultados nacionales y por entidad federativa. Recuperado de https://portalanterior. ine.mx/documentos/Estadisticas2006/presidente/nac.html

31. Instituto Nacional Electoral (INE). (2006). Proceso electoral federal 2006. Recuperado de https://portalanterior.ine.mx/archivos3/portal/historico/contenido/ Proceso_Electoral_Federal_2005-2006/

32. Instituto Nacional Electoral (INE). (2012). Proceso electoral federal 2011-2012. Recuperado de https://portalanterior.ine.mx/archivos3/portal/historico/contenido/ Procesos_Electorales_Acervo_Electronico/

33. Instituto Nacional Electoral (INE). (2018). Cómputos Distritales 2018.

[306] Elecciones Federales. Recuperado de https://computos2018.ine.mx/\#/presidencia/ nacional/1/1/1/1

34. Instituto Nacional de Estadística y Geografía (Inegi). (s. f.). Mortalidad. Conjunto de datos: Defunciones por homicidios. Recuperado de https://www.inegi. org.mx/sistemas/olap/proyectos/bd/continuas/mortalidad/defuncioneshom.asp?s = est

35. Janda, Kenneth. (1980). Political Parties: A Cross-National Survey. New York: Free Press.

36. Krause, Silvana; Kestler, Thomas y Lucca, Juan Bautista. (2013). Los Break-in Parties en América Latina: ¿éxito o fracaso? Revista Debates, 7 (2), pp. 159-171.

37. Kestler, Thomas; Krause, Silvana y Lucca, Juan Bautista. (2017). Cohesión, éxito o fracaso de los nuevos partidos políticos en América del Sur. Revista Mexicana de Estudios Electorales, 1 (18), pp. 195-222.

38. Kitschelt, Herbert. (1994). The Transformation of European Social Parties. New York: Cambridge University.

39. Langston, Joy. (2008). La competencia electoral y la descentralización partidista en México. Revista mexicana de sociología, 70 (3), pp. 457-86.

40. Lago, Ignacio, y Martínez, Ferran. (2011). Why New Parties? Party Politics, 17 (1), pp. 3-20. https://doi.org/10.1177/1354068809346077 
Las elecciones de 2018 en México y el triunfo del Movimiento de Regeneración Nacional...

41. LaPalombara, Joseph y Weiner, Myron. (1966). Political Parties and Political Development. Princeton: Princeton University. https://doi. org/10.1515/9781400875337

42. Levitsky, Steven y Daniel Ziblatt. (2018). How Democracies Die. New York: Crown.

43. Lijphart, Arend. (2000). Modelos de democracia: Formas de gobierno y resultados en treinta y seis países. Barcelona: Ariel.

44. Lipset, Seymour \& Rokkan, Stein (eds.). (1967). Cleavage Structures, Party Systems, and Voter Alignments: An introduction. In: Party Systems and Voter Alignements: Cross National Perspectives (pp. 1-64). New York-London: The Free Press, Macmillan.

45. Lucardie, Paul. (2000). Prophets, Purifiers and Prolocutors: Towards a Theory on the Emergence of New Parties. Party politics, 6 (2), pp. 175-185. https://doi. org/10.1177/1354068800006002003

46. Mainwaring, Scott, Gervasoni, Carlos \& Nájera, Annabella. (2010). The Vote Share of New and Young Parties. Indiana: Helen Kellogg Institute for International Studies.

47. Mair, Peter. (1999). New Political Parties in Established Party Systems: How Successful are They? In: Beukel, Erik; Klausen, Kurt \& Mouritzen, Poul (eds.). Elites, Parties and Democracy: Festschrift for Professor Mogens N. Pedersen, pp. 207-224. Odense: Odense University.

48. Marti i Puig, Salvador y Rovira i Sancho, Guiomar. (2017). Movimientos sociales y acción colectiva. En: Martí i Puig, Salvador; Solís Delgadillo, Juan Mario y Sánchez, Francisco (eds.). Curso de Ciencia Política (pp. 278-218). México, D. F.: Senado de la República.

49. Middlebrook, Kevin. (1988). La liberalización política de un régimen autoritario: el caso de México. En: O’Donnell, Guillermo; Schmitte, Philippe C. y Whitehead, Laurence (comps.). Transiciones desde un gobierno autoritario 2: América Latina. Buenos Aires: Paidós.

50. Modoux, Magali. (2006). Démocratie et fédéralisme au Mexique. Paris: Karthala.

51. Monsiváis-Carrillo, Alejandro. (2018). La izquierda populista en México: ¿amenaza o correctivo para la democracia? Documento de trabajo. El Colegio de la Frontera Norte.

52. Mustapic, Ana María. (2013). Los partidos políticos en la Argentina: condiciones y oportunidades de su fragmentación. En: Acuña, Carlos (comp.). ¿Cuánto importan las instituciones? Gobierno, Estado y actores en la política argentina (pp. 249-290). Buenos Aires: Siglo xxı.

53. Navarrete Vela, Juan Pablo y Espinoza Toledo, Ricardo. (2017). MORENA en las elecciones federales de 2015. Estudios Políticos (México), 40, pp. 71-103. https:// doi.org/10.1016/j.espol.2017.03.001 
54. Olivares, Emir. (2018, febrero 20). Rechaza Anaya acusaciones de triangulación de fondos. La Jornada. Recuperado de https://www.jornada.com.mx/ ultimas/2018/02/20/rechaza-anaya-acusaciones-de-triangulacion-de-fondos-9514. html

55. Panebianco, Angelo. (1990). Modelos de partido: organización y poder en los partidos políticos. Madrid: Alianza.

56. Perkins, Doug. (1996). Structure and Choice: The Role of Organizations, Patronage and the Media in Party Formation. Party Politics, 2 (3), pp. 355-375. https:// doi.org/10.1177/1354068896002003004

57. Rae, Douglas; Hanby, Victor \& Loosemore, John. (1971). Thresholds of Representation and Thresholds of Exclusion: An Analytic Note on Electoral Systems. Comparative Political Studies, 3 (4), pp. 479-488. https://doi. org/10.1177/001041407100300406

58. Ramos Jiménez, Alfredo. (2001). Los partidos políticos latinoamericanos. Mérida: CDCHT, Universidad de los Andes.

59. Sartori, Giovanni. (2000). Partidos y sistema de partidos. Marco para un análisis. Madrid: Alianza.

60. Sánchez Gutiérrez, Arturo. (1988). Los militares en la década de los cincuenta. Revista Mexicana de Sociología, 50 (3), pp. 269-293. https://doi.org/10.2307/3540565

61. Sikk, Allan. (2012). Newness as a Winning Formula for New Political Parties. Party Politics, 18 (4), pp. 465-486. https://doi.org/10.1177/1354068810389631

[308 ] 62. Taagepera, Rein y Shugart, Matthew. (1989). Seats and Votes: The Effects and Determinants of Electoral Systems. New Haven: Yale University.

63. Tavits, Margaret. (2008). Party Systems in the Making: The Emergence and Success of New Parties in New Democracies. British Journal of Political Science, 38 (1), pp. 113-133. https://doi.org/10.1017/S0007123408000069

64. Van Biezen, Ingrid. (2003). Political Parties in New Democracies: Party Organization in Southern and East-Central Europe. New York: Palgrave Macmillan. https://doi.org/10.1057/9781403937858

65. Vice, Margaret \& Chwe, Hanyu. (2017). Mexican Views of the U.S. Turn Sharply Negative. Pew Research Center. Recuperado de http://www.pewglobal. org/wp-content/uploads/sites/2/2017/09/Pew-Research-Center_09.14.17_MexicoReport.pdf

66. Villamil, Jenaro. (2012). Peña Nieto: el gran montaje. México, D. F.: Grijalbo. 